\title{
Quantum Computation in Quantum-Hall Systems
}

\author{
V. Privman, ${ }^{1}$ I. D. Vagner ${ }^{2}$ and G. Kventsel ${ }^{2,3}$ \\ ${ }^{1}$ Department of Physics, Clarkson University, Potsdam, \\ New York 13699-5820, USA \\ ${ }^{2}$ Grenoble High Magnetic Field Laboratory, Max-Planck-Institut für \\ Festkörperforschung, and Centre National de la Recherche \\ Scientifique, BP 166, F-38042, Grenoble Cedex 9, France \\ ${ }^{3}$ Department of Chemistry, Technion-Israel Institute of Technology, \\ Haifa 32000, Israel
}

\begin{abstract}
We describe a quantum information processor (quantum computer) based on the hyperfine interactions between the conduction electrons and nuclear spins embedded in a two-dimensional electron system in the quantum-Hall regime. Nuclear spins can be controlled individually by electromagnetic pulses. Their interactions, which are of the spin-exchange type, can be possibly switched on and off pair-wise dynamically, for nearest neighbors, by controlling impurities. We also propose the way to feed in the initial data and explore ideas for reading off the final results.
\end{abstract}

Submitted to Physics Letters A 
The field of quantum computing has seen an explosive growth of theoretical development [1-7]. It has been realized that quantum computers can be faster than classical computers for some problems [1-3,8-13]. The analog nature of errors and possible error correction schemes have been explored $[6,7,9,13-21]$. There have also been several proposals for actual realizations of quantum information processing [4,5,13,22-31]. Two of these proposals: the ion-trap system $[5,22,25,27,28]$ and the ensemble-of-molecules liquid-state NMR approach [29-31] have been studied extensively as possible experimental realizations of quantum computing. However, all experimental results to date only accomplish the simplest quantum-logic functions such as single-spin rotations or two-spin controlled-NOT [1-7].

A major challenge faced by both experiment and theory has involved scaling up from one to many quantum gates and actual "programming," i.e., conducting calculations by coherent quantum unitary evolution, in a controlled fashion. Experimentally, quantum computation requires switching on and off pair-wise interactions between various two-state systems, e.g., spins $\frac{1}{2}$, termed "qubits." Initialization and reading off the final results are also nontrivial parts of the process. Ideally, the latter should involve efficient measurement of a single qubit. The NMR variant [29-31] measures instead ensemble averages (expectation values). Certain "fault-tolerant" error correction schemes $[7,13,17,19-21]$ actually also require measurements of some of the qubits during the computation.

Theoretically, the most striking recent development has been the formulation of the fault-tolerant error correction schemes [7,13,17,19-21]. Correction of analog errors inherent in quantum computation due to the superpositionof-states property (which in turn is central to the speed-up of some calculations) means an uphill battle against the second law of thermodynamics. These error-correction schemes $[7,13,17,19-21]$ aim at calculations that can go on indefinitely provided the overall error rate at each qubit is small enough.

It is not our goal here to review these issues: we will adopt the point of view that modern error correction schemes will allow calculations long enough to be useful provided a working quantum information processor can be devised. It is the latter aspect that we address in this work. Thus, we propose a quantum computer realization based on hyperfine interactions [32] between the conduction electrons and nuclear spins embedded in a twodimensional electron system in which the electron gas is in the quantum-Hall effect (QHE) regime [33,34]. Such systems have been made at the interfaces 
between semiconductor materials and in superlattices (layered semiconductor structures) [35].

In these systems, at temperatures of order $1 \mathrm{~K}$ and applied magnetic fields of several Tesla, there are intervals of magnetic field values for which the electrons fill up an integer number of Landau levels [36]. The electron gas then forms a nondissipative QHE fluid [35]; the Hall resistance exhibits a plateau at a value that is a multiple of $e^{2} / h$, while the dissipativity of the conduction electron gas (the magnetoresistance) approaches zero. Nuclearspin thermalization/relaxation processes occur on the time scale denoted $T_{1}$ [32] which, experimentally, ranges from several minutes to half an hour [37-39]. It is expected then that the nuclear spin dynamics is dominated by coherent spin exchanges mediated by electrons $[40,41]$. Owing to rapid advances in the experimental facilities, the hyperfine interactions in QHE systems have recently attracted growing theoretical $[41,42]$ and experimental [37-39] interest; this progress makes it feasible to handle the electron spinnuclear spin interactions with almost atomic precision.

Similar to the ion-trap system $[5,22,25,27,28]$, we consider a chain of spin$\frac{1}{2}$ nuclei, of atoms positioned by the molecular-beam epitaxy techniques [35] in an effectively two-dimensional system subjected to a strong magnetic field. The typical separation should be comparable to the magnetic length $\ell_{H}=$ $\sqrt{\hbar c / e H}$, where $H$ is the applied magnetic field, perpendicular to the twodimensional layer. This length is of the order of $100 \AA$. We propose to control individual nuclear spins by electromagnetic-radiation pulses in the nuclear magnetic resonance (NMR) frequency range [32].

An important question is how to control nuclear spins individually. Use of magnetic field gradient could be contemplated to achieve differentiation, but there are severe limitations on the field variation owing to the need to maintain the QHE electronic state. Instead, one can use different nuclei. Theoretically, there is no apparent limit on how many different spins can be arranged in a chain. However, practically the number of suitable spin- $\frac{1}{2}$ isotopes may be limited. Thus, achieving sufficient chemical-shift dispersion for systems of more than few qubits may require additional ideas; the following ones are tentative because presently it is not known how realistic these proposals are from the point of view of actual experimental realizations. Specifically, one can position nuclear spins in different crystalline environments. The latter can be controlled by implanting atoms and complexes into 
the host material [32]. It may be also possible to utilize small clusters of nuclear spins, rather than individual spins. These can be made coherent [43] by lowering the temperature to order several $\mu \mathrm{K}$, as compared to order $1 \mathrm{~K}$ needed to achieve the QHE state.

Under the typical conditions of QHE the direct dipole-dipole interaction of the nuclear spins is negligibly small [41]. The dominant interaction will be that mediated by the contact hyperfine interactions between nuclear spins and conduction electrons [40]. Similarly, electron-mediated interactions leading to the scalar coupling have been utilized in the liquid-state NMR realization of quantum computation [29-31]. In ordinary metals, the electron-mediated nuclear-spin interactions exhibit Friedel oscillations [32] because of the existence of sharp Fermi surface.

In the quantum-Hall regime, however, the energy spectrum of the twodimensional electron gas is discretized by the magnetic field. As a result, the interaction is no longer oscillatory but rather monotonic, exponentially decaying [40] on the length scale $\ell_{H}$. The following terms in the effective phenomenological two-spin interaction Hamiltonian correspond to the secondorder perturbative calculation (carried out for two identical nuclei) of [40], where for different nuclei we replaced $Z^{2}$ by the product of the two atomic numbers (which is basically a guess),

$$
-V Z^{(1)} Z^{(2)} H^{-1} \sqrt{\frac{c \ell_{H}}{r}} e^{-c r / \ell_{H}}\left[\sigma_{-}^{(1)} \sigma_{+}^{(2)}+\sigma_{+}^{(1)} \sigma_{-}^{(2)}\right],
$$

where $c$ is a dimensionless quantity [40] of order $1, Z^{(j)}$ are the atomic numbers of the nuclei, while $V$ is some constant. Note that

$$
r / \ell_{H} \propto r \sqrt{H}
$$

Here $H$ is the applied field, $r$ is the spin-spin separation, while $\sigma^{(j)}$ are the Pauli matrices corresponding to the spin- $\frac{1}{2}$ operators of the two nuclei labeled by the superscripts $j=1,2$. Each nuclear spin also interacts with the applied field via the magnetic coupling of the form $-\gamma^{(j)} \hbar H \sigma_{z}^{(j)}$. Determination of the precise effective spin-spin interaction Hamiltonian will likely to be accomplished to a large extent by direct experimental probe. The strength of the interaction in Eq. (1) can be roughly estimated to be of order $10^{-16} \mathrm{erg}$, which corresponds to frequency of order $10^{11} \mathrm{~Hz}$. 
For quantum computation, one has to devise the means to control the spin-spin interactions. Ideally, one would like to be able to switch interactions on and off at will, for varying time intervals $\Delta t$. Switching on a pair-wise interaction would allow to carry out a unitary transformation on a pair of spins independently of the other spins. It has been established [13,23,44-47] that nearly any such transformation, combined with single-spin transformations which can be accomplished by radiation pulses, form a universal set in the sense that arbitrary "computer program" can be built from them. There are NMR "refocusing" methods that allow such control, as utilized, for instance, in the liquid-state NMR formulation [29-31] of quantum computing. However, until the full form of the spin-spin interaction Hamiltonian is established for our case, it is useful to consider other ideas as well.

Geometry constraints would limit the pairs of spins for which the two-spin interactions are nonnegligible typically to nearest-neighbor pairs. Furthermore, other interactions cannot be really fully eliminated, but only reduced. Still, control of the spin-spin interactions would allow added flexibility in "programming" the unitary evolution of a computational device. Even when the control is possible, in practice it would be unrealistic to expect the form of the interaction, such as Eq. (1) above, be known exactly from theoretical calculations alone. Thus, Eq. (1) is a leading-approximation/guess phenomenological form. Input from experiments will be required to fine-tune the computer functions that depend on such internal interactions.

One possibility not based on the NMR methods is to disrupt (ideally, switch off), for the duration of some time interval $\Delta t$, the interaction for one (nearest-neighbor) pair of spins by placing impurities between the spins, see Figure 1. The impurities can be ionized by external electromagnetic pulses to electronic configurations that capture electrons and locally destroy the coherence of the electron gas. Differentiation can be achieved by using different impurity species. Admittedly, this is a rather speculative idea. Specifically, it may be more appropriate to place the impurities near or surrounding the nuclear spins, instead of the geometry of Figure 1.

It is important to emphasize that the pair-wise interactions are "on" most of the time, for each pair of spins. Therefore, the "idle" unitary transformations in the latter approach will not be simple phase changes as for noninteracting spins. The ability to change the interactions locally, pair-wise, will only allow to change the relative unitary transformations to which nearestneighbor spin pairs are subject. In addition, one has the single-spin rotations 
that can be done by external electromagnetic pulses. Programming of such a computer is therefore less straightforward than usually expected in the theoretical approaches that assume noninteracting idling elements $[1-7,13,23,44$ 47]; however, this is only a matter of new mathematical developments being called for.

We now turn to the process of "feeding in" the initial data into the computer. This can be accomplished as follows: initially, all the nuclear spins in the system are pumped in one direction. This can be achieved by shining a polarized light at the system [49] that creates electron-hole pairs. These pairs annihilate, forcing on a fixed nuclear spin polarization, corresponding to that of the incident light [49]. After the initial alignment, the nuclear spins can be rotated to the desired quantum states needed for computation by electromagnetic pulses at their respective frequencies.

In all the proposals for quantum computation [1-7,13,22-31], reading off the final spin states by measuring, and also the measurement processes that are required for error correction [7,13,17,19-21], are most challenging to realize. This is because direct interaction of a microscopic system with any macroscopic system for the purpose of measurement is disruptive and difficult to carry out in an orderly fashion for all the individual spins in the system.

We note that as for the liquid-NMR proposal [29-31], we could read off averages by NMR techniques by producing replicas of the spin chain, see Figure 1, and letting them evolve in parallel. The electromagnetic pulses that control the computation can be applied to all the replicas at once. However, some quantum error correction protocols $[7,13,17,19-21]$ require actual measurements rather than averages. Furthermore, unlike the liquid-state NMR, there may be uncontrollable differences between the replicas. The only thing that might save the situation is the fact that our spins are located at distances much larger than atomic dimensions. Therefore, some averaging of the "atomic" scale influences may be expected in the spin-spin interactions controlling the actual computation in each chain. The latter observation suggests that measurement methods other than NMR-based must be explored. We propose three measuring processes below: the first and second may be more appropriate for final-state readout while the second and third for errorcorrection schemes.

First, let us assume that the final state is one of the direct-product states of the $n$-spin system. It is possible to generate by holographic and other 
methods [48-51] a narrow strip of conductance at each spin in turn, see Figure 1, and send a current of spin-polarized electrons through it. The observed current can be pre-calibrated to enable high certainty determination of whether there was a spin-exchange scattering event thus determining the nuclear spin's direction, resembling the spin-diode $[38,52]$ techniques. Furthermore, one can extend the strip of conductance over several replicas of the spin chain, separated order of magnitude more than the spins, e.g., $1000 \AA$. One can probably have enough of them to reduce significantly any uncertainty in the spin direction determination.

Second, if the final or intermediate state (the latter case is relevant for error correction) can be entangled, so that one cannot simply measure each spin in turn, then the situation is more complicated. One can generate a "mask" of conducting strips, for all or a group of spins. However, "calibration" to derive data pertinent to the multispin quantum state may be a challenge.

Third, some error correction schemes [7,13,17,19-21] require measurement of difference of the components of nearby spins. This might be contemplated by having two conducting strips with the spin-polarized electron current, and adding a time-dependent component to the applied magnetic field for the duration of the measurement. Difference in the nuclear spin states will then affect the Aharonov-Bohm oscillatory structure of the observed current; see [53] for survey of such effects.

In summary, we have proposed a model of a quantum computer based on the hyperfine interactions between the electron and nuclear spins in quantum Hall effect systems. This brings to two the number of proposals that have been formulated theoretically for realizations of quantum computing which can be potentially done in solid-state systems; the other is the quantum-dot proposal [26]. The possibility of quantum computing in solid-state is exciting. Indeed, the intricacies of modern technology, especially as far as nanoscale "engineering" is concerned, are much more geared for solid-state systems than any other medium. All modern electronic devices, with, presently, components on submicron scales, are solid-state.

However, unlike the more "established" quantum computing proposals such as ion traps and liquid-state NMR, the two solid-state proposals are presently theoretical. There are several investigations needed, of the form and strength of the spin-spin interactions, of the time scales of interaction vs. decoherence, and other topics, before initial experimental attempts to 
build few-qubit QHE quantum-computing systems can be deemed realistic. Specifically, no estimates are available of the time scales of decoherence which may be orders of magnitude shorter than $T_{1}$.

We wish to thank D. Mozyrsky for helpful comments on the manuscript and P. Wyder for the hospitality at Grenoble HFML and interest in this work. The work of V.P. has been supported in part by US Air Force grants, contract numbers F30602-96-1-0276 and F30602-97-2-0089. I.V. acknowl-

edges support by a grant from the German-Israeli Foundation for Scientific Research and Development, number G 0456-220.07/95.

\section{References}

[1] C.H. Bennett, Physics Today, October 1995, p. 24.

[2] D.P. DiVincenzo, Science 270, 255 (1995).

[3] A. Ekert and R. Jozsa, Rev. Mod. Phys. 68, 733 (1996).

[4] S. Haroche and J.-M. Raimond, Physics Today, August 1996, p. 51.

[5] B. Schwarzschild, Physics Today, March 1996, p. 21.

[6] D. Mozyrsky, V. Privman and S. P. Hotaling, in SPIE Conf. AeroSence 97, Proc. no. 3076 (SPIE Publ., 1997).

[7] J. Preskill, Reliable Quantum Computers, preprint (available at http://xxx.lanl.gov/abs/quant-ph/9705031).

[8] P. Shor, Algorithms for Quantum Computation: Discrete Logarithms and Factoring, in Proc. 35th Ann. Symp. on Fundamentals of Computer Science (1994).

[9] I. L. Chuang, R. Laflamme, P. W. Shor and W. H. Zurek, Science 270, 1633 (1995).

[10] C. Dürr and P. Høyer, A Quantum Algorithm for Finding the Minimum, preprint (available at http://xxx.lanl.gov/abs/quant-ph/9607014).

[11] R.B. Griffiths and C.-S. Niu, Phys. Rev. Lett. 76, 3228 (1996). 
[12] L.K. Grover, A Fast Quantum Mechanical Algorithm for Estimating the Median, preprint (available at http://xxx.lanl.gov/abs/quant$\mathrm{ph} / 9607024)$.

[13] D.P. DiVincenzo, Topics in Quantum Computers, preprint (available at http://xxx.lanl.gov/abs/cond-mat/9612126).

[14] R. Landauer, Philos. Trans. R. Soc. London Ser. A 353, 367 (1995).

[15] P. Benioff, J. Stat. Phys. 29, 515 (1982).

[16] A. Peres, Phys. Rev. A 32, 3266 (1985).

[17] E. Knill and R. Laflamme, A Theory of Quantum Error-Correcting Codes, preprint (available at http://xxx.lanl.gov/abs/quant-ph/9604034).

[18] A. Ekert and C. Macchiavello, Phys. Rev. Lett. 77, 2585 (1996).

[19] P. Shor, Fault-tolerant quantum computation, preprint (available at http://xxx.lanl.gov/abs/quant-ph/9605011).

[20] D. Gottesman, Stabilizer Codes and Quantum Error Correction, preprint (available at http://xxx.lanl.gov/abs/quant-ph/9705052).

[21] D. Aharonov and M. Ben-Or, Fault-Tolerant Quantum Computation with Constant Error, preprint (available at http://xxx.lanl.gov/abs/quant-ph/9611025).

[22] J. I. Cirac and P. Zoller, Phys. Rev. Lett. 74, 4091 (1995).

[23] D. P. DiVincenzo, Phys. Rev. A 51, 1015 (1995).

[24] S. P. Hotaling, Radix-R $>2$ Quantum Computation, preprint (1996).

[25] A. Steane, The Ion Trap Quantum Information Processor, preprint (available at http://xxx.lanl.gov/abs/quant-ph/9608011).

[26] D. Loss and D. P. DiVincenzo, Quantum Computation with Quantum Dots, preprint (available at http://xxx.lanl.gov/abs/condmat/9701055). 
[27] C. Monroe, D. M. Meekhof, B. E. King, W. M. Itano and D. J. Wineland, Phys. Rev. Lett. 75, 4714 (1995).

[28] Q. Turchette, C. Hood, W. Lange, H. Mabushi and H. J. Kimble, Phys. Rev. Lett. 75, 4710 (1995).

[29] N. A. Gershenfeld and I. L. Chuang, Science 275, 350 (1997).

[30] D. G. Cory, A. F. Fahmy, T. F. Havel, in PhysComp 96, eds. T. Toffoli, M. Biafore and J. Leao (New England Complex Systems Inst. 1996).

[31] D. G. Cory, A. F. Fahmy, T. F. Havel, Proc. Nat. Acad. Sci. 94, 1634 (1997).

[32] C. P. Slichter, Principles of Magnetic Resonance, Third Ed. (SpringerVerlag, Berlin, 1996).

[33] K. von Klitzing, G. Dorda and M. Pepper, Phys. Rev. Lett. 45, 494 (1980).

[34] D. C. Tsui, H. L. Störmer and A. C. Gossard, Phys. Rev. Lett. 48, 1559 (1982).

[35] R. E. Prange and S. M. Girvin, The Quantum Hall Effect (SpringerVerlag, Berlin, 1990).

[36] L. D. Landau and E. M. Lifshits, Quantum Mechanics. Nonrelativistic Theory (Nauka, Moscow, 1974).

[37] A. Berg, M. Dobers, R. R. Gerhardts and K. von Klitzing, Phys. Rev. Lett. 64, 2563 (1990).

[38] K. Wald, L. P. Kouwenhoven, P. L. McEuen, N. C. Van der Vaart and C. T. Foxon, Phys. Rev. Lett. 731011 (1994).

[39] R. Tycko, S. E. Barret, G. Dabbagh, L. N. Pfeiffer and K.W. West, Science 268, 1460 (1995).

[40] Yu. A. Bychkov, T. Maniv and I.D. Vagner, Solid State Commun. 94, 61 (1995). 
[41] I. D. Vagner and T. Maniv, Physica B 204, 141 (1995).

[42] I. D. Vagner and T. Maniv, Phys. Rev. Lett. 61, 1400 (1988).

[43] M. Goldman, Spin Temperature and Nuclear Magnetic Resonance (Clarendon Press, Oxford, 1970).

[44] A. Barenco, Proc. R. Soc. Lond. A 449, 679 (1995).

[45] A. Barenco, Contemporary Phys. 37, 375 (1996).

[46] A. Barenco, C. H. Bennett, R. Cleve, D. P. DiVincenzo, N. Margolus, P. Shor, T. Sleator, J. A. Smolin and H. Weinfurter, Phys. Rev. A 52, 3457 (1995).

[47] S. Lloyd, Phys. Rev. Lett. 75, 346 (1995).

[48] R. W. Winkler, J. P. Kotthans and K. Ploog, Phys. Rev. Lett. 62, 1177 (1989).

[49] D. Weiss, K. von Klitzing, K. Ploog and G. Weimann, Europhys. Lett. 8, 179 (1989).

[50] R. R. Gerhardts, D. Weiss and K. von Klitzing, Phys. Rev. Lett. 62, 1174 (1989).

[51] Yu. A. Bychkov, T. Maniv, I. D. Vagner and P. Wyder, Phys. Rev. Lett. 73, 2911 (1994).

[52] B. E. Kane, L. N. Pfeiffer and K. W. West, Phys. Rev. B 467264 (1992).

[53] I. D. Vagner, A. Rozhavsky, A. Zyuzin and P. Wyder, Is the Magnetic Field Necessary for the Aharonov-Bohm Effect in Mesoscopics?, preprint (1997). 


\section{FIGURE CAPTION}

Figure 1: The schematics of the proposed two-dimensional nuclear-spin system: N denotes atoms with spin- $\frac{1}{2}$ nuclei; I denotes impurity atoms or complexes that can be ionized to disrupt the spin-exchange interactions mediated by conduction electrons (the impurity placement may be different, see text); $\mathrm{R}$ illustrate replicas (actually there will be many of them); E and C represent conducting electrodes and connecting strip for measurement (see text). 


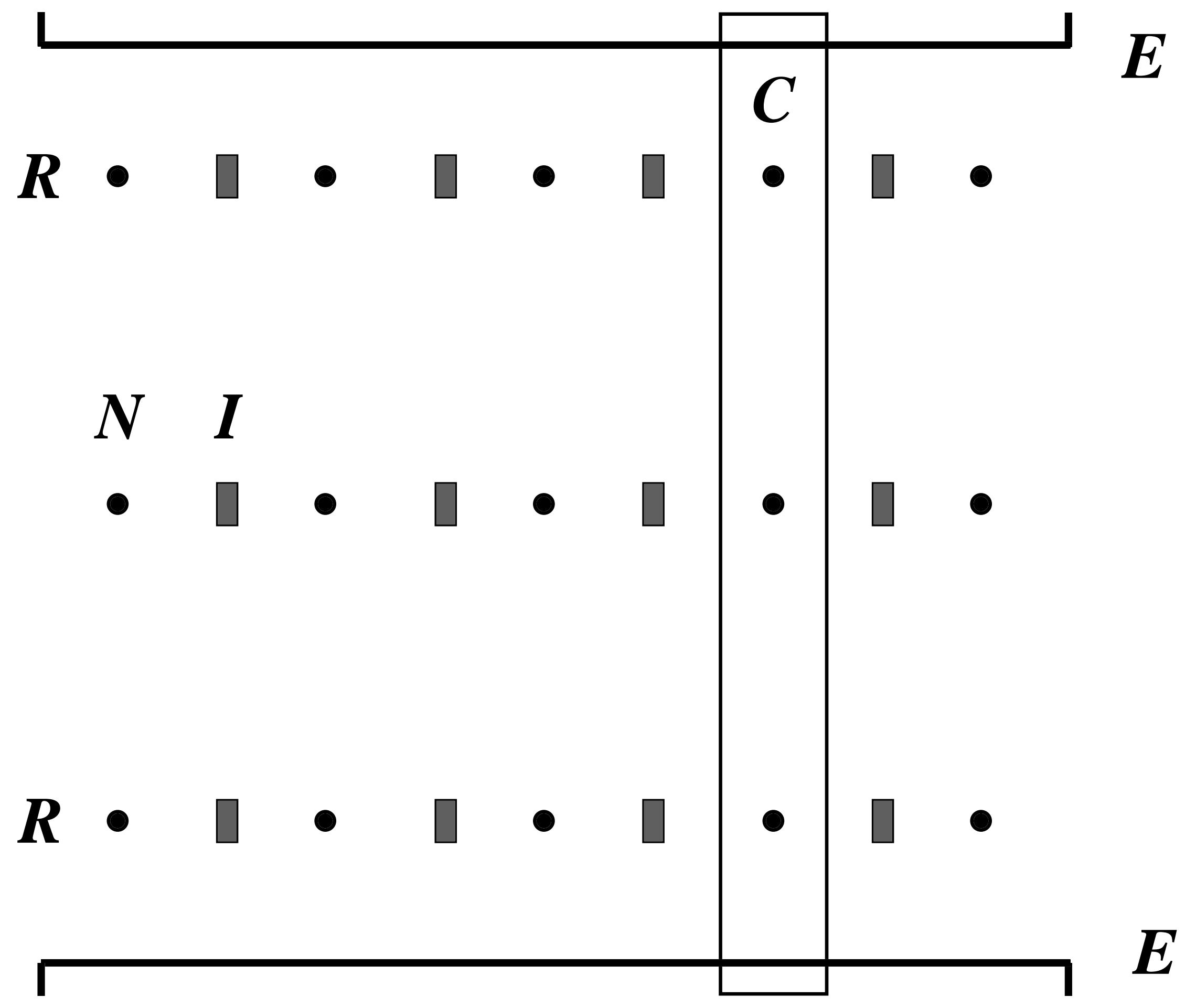

